\title{
Interactive learning as an "emerging" technology: A reassessment of interactive and instructional design strategies
}

\author{
Roderick C.H. Sims \\ Southern Cross University
}

\begin{abstract}
This paper challenges the assumptions and accepted practices used in the design and development of interactive learning resources. Through an assessment of accepted assumptions and the pitfalls inherent in instructional design it is debated whether technology can effectively replicate the adaptability and flexibility of human communication. It is proposed that interactive technology is only now emerging as a viable alternative, requiring not only a new approach to the presentation of interactive materials but also an integrated methodology which is relevant to the tools and practices of today.
\end{abstract}

\section{Introduction}

Can learning and performance truly be enhanced through technology? This question can only be answered if we step aside and assess the critical components in the field of educational multimedia. Over the past 25 years, new developments in technology have regularly prompted advertisements or editorial comment which predict a world of educational utopia and true individualised learning. In the late 1990s, we continue to be confronted with educational technology solutions, this time in the form of multimedia, the internet and web-based learning; and many opportunists have been quick to implement applications incorporating these technologies. Despite the various reports which have debated the effectiveness of traditional interactive technology applications (Roblyer, 1988; Sims \& Schwalger, 1992), the validity of research into educational technology (Reeves, 1993) as well as the appropriateness or relevance of Instructional Design methodologies (Gillespie, Sims \& Spannaus, 1992; Merrill, 1997), the latest exemplars often lack the structures or strategies proven to be effective for learning. 
Rather than focus on new technologies and their potential for education and training, this paper analyses fundamental elements of educational technology to assess whether the current approaches used in the design and development of interactive learning applications are valid. The analysis initially considers a selection of assumptions relevant to interactive learning and identifies factors which contradict their validity. This is followed by a review of traditional Instructional Systems Development (ISD) methodologies to provide a background for the introduction of an enhanced model designed specifically to support the development of effective interactive learning applications.

\section{Assumptions of interactive learning}

While there has been little debate on the instructional function of resources such as books, study guides or lectures, no such consensus has been established for interactive learning materials. There appears to be no common set of standards for the learner-computer interface, the strategies for presentation of interactive material is variable and the influx of newer technologies (such as multimedia and web-based resources) have only added to the complexity of developing effective interactive learning materials (Viers, 1996). Given this situation, it is important to assess the assumptions which are inherent in both research analyses and courseware applications to determine their validity for educational technology.

Based on research and courseware development projects over the past 15 years (e.g. Sims, 1990; 1996), a set of assumptions can be identified which provide a framework for the development of effective interactive learning materials. Even so, many observers continue to be critical of the poor quality of courseware observed in the marketplace (e.g. Roblyer, 1988; Trollip, 1992; Foshay, 1997). As a challenge to the interactive learning discipline and the very nature of educational technology, these assumptions will be critically examined. It will be shown that we do not yet have a good understanding of learner-computer interactions and the communication dynamics between learner and computer, and that the instructional strategies we adopt must be suitable for the technology, rather than the technology being used to imitate traditional instructional techniques.

\section{Assumption 1: Technology makes learning more effective}

A common assumption in general educational literature is that interaction and communication between and among participants in the learning process is critical to achieving outcomes. Acceptance of this first assumption implies that technology has an inherent benefit over other educational 
resources and that only through extensive developments in technology will effective interactive instruction become a reality. Typical of this attitude is a recent report on a web-based learning initiative which concluded that "the Internet has overtaken the CD-ROM as the cheapest, fastest and most interactive mode of computer-based learning" (The Australian, 1997:47). While this technological evolution holds true, it does not determine whether the Internet is more effective as an educational resource. While early studies of CAI assessed through meta-analysis reported technologybased instruction to be at least as effective as traditional instruction (e.g. Kulik, Kulik \& Cohen, 1980), the validity of the research has been queried (Reeves, 1993) and other commentators have debated the level of effectiveness (Clark, 1983; Sims, 1992). Even 10 years ago, Roblyer (1988:7) suggested:

it is becoming more difficult to make a case for increased across-the-board implementation of CAI on the basis of research results. A review of past CAI uses ... indicates that effects on learning vary widely depending on product design and implementation, and that CAI may often not be as effective in raising student performance as other, less expensive nontraditional methods.

Response to such observations have prompted calls for more stringent design principles to be applied to courseware development activities (for example, the on-going discussions on ITFORUM ${ }^{1}$ ). However, it is possible that the limited effectiveness has resulted from a poor understanding of the technology for interactive learning, rather than design integrity and that effectiveness will only be achieved when we fully understand the functionality and vagaries of the interactive technology itself.

Practitioners in the field of interactive technology are regularly faced with the dilemma of whether to commence development or wait for the next technological advance, and recent developments in multimedia, virtual and internet systems are encouraging the development of applications with realistic, situate or contextual environments (Tessmer \& Richey, 1997). The implication is that the closer interactive learning comes to reality the better it will be, and that new technologies provide more individualised options (Larsen, 1992). However, as Tessmer \& Richey (1997:85) note "Context is a pervasive and potent force in any learning event. Yet instructional design models contain little guidance about how to accommodate contextual elements to improve learning and transfer" which

\footnotetext{
${ }^{1}$ ITFORUM is an internet-based discussion group (listserve) focusing on Instructional Technology and may be accessed by subscribing to LISTSERV@UGA.CC.UGA.EDU.
} 
reinforces the need to develop instructional design models specific to the technology. Technology is not the solution for effective learning, but an understanding of interactive technology and how it can support education and learning is essential, as our social infrastructure increasingly relies on that technology for communication and information transfer.

\section{Assumption 2: Traditional teacher-student interactions can be mapped directly to interactive learning}

Acceptance of this assumption implies that the strategies and practices used in traditional teacher-learner interactions (such as the classroom) can be equally functional and effective when computer-delivered. Since interactive technology was introduced as a potential resource for education and training, it has been assumed that the methods and approaches traditionally used for learning would map exactly to the technology (e.g. the Tutorial, Drill, Game, Test and Simulation modes identified by Alessi \& Trollip, 1991). However, as many applications continue to be criticised (Foshay, 1997), then it must be argued whether interactive technology is in fact a viable medium for instructional strategies, or if new approaches and techniques will be required to bring the technology to its potential as a learning resource.

This assumption forms the basis of most interactive training technology. However, if we have not yet unravelled the true functionality of interactivity (Sims, 1997), then attempts to replicate traditional resources may be misdirected. In fact, the very selection of tutorial as an instructional strategy (one of the most common) may be at fault, because technology may not necessarily be suitable as a presenter of instructional material. Therefore, one of today's challenges, with what can still be regarded as an emerging technology, is to assess the best means by which instructional material should be presented to the learner, and to what extent technology may be most appropriate.

\section{Assumption 3: Interactive learning will cater for individual differences and learning styles}

Acceptance of this assumption implies that courseware can be developed which can respond to the individual needs of learners, and that this is a desirable use of the technology. Jonassen (1988:202) suggests that "the power of interactive technologies ... lies in their ability to adapt instruction in ways that make it more meaningful" and Ross \& Morrison (1988:227) claim that "one of the computer's most powerful capabilities lies in 
adapting instruction to students". However, the power of the interaction has yet to be fully explored, as suggested by Sims (1997).

While there is no doubt that complex interactions and branching structures can be produced based on learner responses, the assumption remains that this is an appropriate form of presentation. However, the issue is that little is known about the way people learn with technology, and the creation of complex interactions may not accurately adapt to their individual needs. This argument extends to the implementation of intelligent tutoring systems, which attempt to generate material based on student performance. While superficially desirable, it is unclear whether technology can effectively replicate the adaptability and flexibility of human communication. Similarly, there is the assumption that artificial intelligence is superior as an instructional technology. However, it is unclear how students perceive such intelligent interactions and responses, and whether such artificial elements are more effective educationally. For effective learning and adaptation to the individual, it will be essential to address issues relating to the extent to which technology might support new modes of human communication and whether interactive learning environments will accommodate rather than adapt to variations in learning styles and individual differences.

Is technology simply a means to replicate human interaction in an educational context, or do we have a new medium for educational communication which therefore demands alternative methods of design and understanding?

\section{Assumption 4: Design and development methodologies will improve courseware quality}

Acceptance of this assumption implies that the methodologies currently promoted are valid for interactive technology and that Instructional Systems Development (ISD) techniques implemented by experienced instructional designers will produce effective courseware. While ISD is well established, its foundation is in traditional instructional applications rather than interactive technology; therefore, it may be that models and procedures specifically designed for interactive applications will be more effective (for example: Yang, Moore \& Burton, 1995; DeWeaver \& Gillespie, 1997). As the techniques and tools for developing interactive applications become more sophisticated, it is likely that the rigorous nature of ISD methodologies will be replaced by more flexible techniques. 


\section{And if the assumptions are wrong?}

This brief analysis has provided a critique of selected assumptions associated with interactive learning. If these generally accepted options for interactive learning are invalid, then new models and concepts will be required to enable educational technology to reach its potential. The following discussion focuses on a variation to the traditional instructional design models and introduces enhanced techniques and options designed specifically to take advantage of interactive technology.

\section{Instructional design}

Traditional Instructional Systems Development (ISD) methodologies present a linear approach to materials creation, with one set of activities logically following another (Logan, 1979:1):

Instructional systems development is ... a general systems approach ... used to produce an instructional system. The phases are sequential sets of activities called analysis, design, development, implementation and control.

Many researchers have developed models and methodologies for the development of instruction, and the common characteristics of these models are the sequential phases of Analysis, Design, Development, Implementation and Evaluation (as summarised by Gustafson, 1991). More recent work by Leshin, Pollock \& Reigeluth (1992) reinforce this sequential approach, although the need for an additional focus on both tactics and strategies is included. Significant research has also been completed by M. David Merrill and colleagues in developing the concept of second generation instructional design (Merrill, Li \& Jones, 1990) and the notion of instructional transactions which provide prescriptive shells to assist developers in selecting the correct match of presentation strategy, learner and content (Merrill, Li \& Jones, 1991). This has recently been extended to include the notion of knowledge objects (Merrill, 1997) where the strategy and knowledge associated with that strategy are separated, enabling a range of instructional prescriptions depending on the current learning task.

These developments in instructional design theory provide important information about the relationship between content and presentation, although the transfer and transition to interactive learning materials continues to assume the suitability of technology as a presentation device. When used by experienced practitioners, ISD provides a reliable set of 
procedures for structuring and sequencing instructional material. However, the very prescriptive nature of ISD can result in technically correct structures which focus on the content of the subject matter rather than its use in an interactive performance environment. Given ISD's capability to structure material for instruction, the question remains as to whether educational technology is a suitable medium for ISD generated materials.

To cater for the successful design, development and implementation of interactive instructional materials an expanded model is proposed which extends the essential elements of ISD methodologies and integrates project management and quality control practices, relating to the recent work by Phillips (1996). As detailed below, this model provides a flexible approach to courseware development based on real-world experiences.

\section{The Interactive Instruction Influence Development Model}

The Interactive Instructional Influence Development $\left(\mathrm{I}^{3} \mathrm{D}\right)$ model proposed by Aubrey (1992) has its basis in practical software development and quality control experiences, and incorporates both instructional design and project management. The concept integral to the model, influence, makes it significantly different from ISD models by specifying that each major activity has varying levels of influence throughout the project. While this was impractical when programming languages and mainframe computer networks provided the delivery medium, the availability of desktop applications and prototyping tools makes the development of interactive learning a more flexible activity (Allen, 1992). $I^{3} \mathrm{D}$ provides significant flexibility for the developer and project manager, as well as being directly relevant to the tools and techniques available to the courseware developer. While the structure of the model differs from the traditional sequential ISD format, the components necessary for effective design and development are integrated within the model.

The $I^{3} \mathrm{D}$ structure (illustrated in Figure 1) is designed to include all of the major functions associated with courseware development - Deliverables, Techniques and Skills. The concept behind the model is that both the techniques and skills associated with interactive materials development have influence throughout the project, with the extent of influence represented by the angles forming the apex of each triangle. Where a technique or skill has constant influence throughout the project, it is represented by a vertical bar. By viewing the model as a whole, the complete process of interactive materials development can be understood, with the relative influence of each stage in the overall project visually 
portrayed. To provide additional information on the $I^{3} \mathrm{D}$ model, a brief description of the various functions follows.

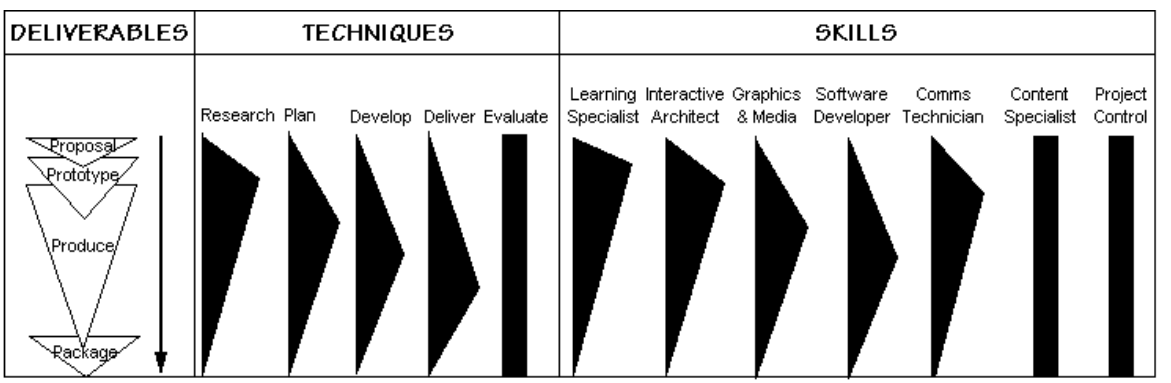

Figure 1: The Interactive Instructional Influence Development Model

\section{Deliverables}

The deliverables represent those components which must be achieved and verified by the project manager - items which must be presented to the client at certain stages of the project. Rather than viewing courseware development as a series of phases (analysis, design, development, implementation, evaluation), it may be more appropriate to view the project as four discrete yet interconnected deliverables. The representation of each deliverable as overlapping is designed to indicate the need for a smooth transition from project start to completion. Depending on the nature and scope of the project, the apex of each deliverable triangle may be extended or reduced to cater for project specifics.

In the current development environment, this becomes especially significant as the difference between prototype and production model is often impossible to qualify. In addition, the tendency to focus on learnercentred development which includes members of the target audience in the production team implies that the prototype will determine major operational parameters of the final product. The following summary provides a brief description of the major elements in the Deliverables component of the $\mathrm{I}^{3} \mathrm{D}$ model:

\section{- Proposal}

The proposal, preferably including exemplar or prototype courseware, provides documentation which describes the overall nature of the project, and conditions to which the client will agree. The $I^{3} \mathrm{D}$ model provides the flexibility for the proposal to be finalised while formal prototyping is being completed. In terms of the associated techniques and skills, it is likely that 
design and development work will be required to ensure the client has a clear idea of the look-and-feel of the product. However, the importance of understanding expectations between client and developer cannot be understated.

\section{- Prototype}

The second major task for an interactive learning project involves prototyping, where potential components for the product are tested and refined, typically using a technique of successive approximations (Allen, 1992). With the tools now available for developing interactive products (both desktop and on-line), prototyping has become a critical component of the overall project, and may form a major part of the actual production effort.

\section{- Production}

The major activity of an interactive project will focus on the production of the interactive application and supportive materials. This task links closely with the prototyping, and continues until the product is ready for packaging and release. Given the recent trend towards a spiral approach to interactive software development (e.g. DeWeaver \& Gillespie, 1997), a consideration for developers will be to merge the prototype and production tasks into a single activity.

\section{- Package}

The final task associated with the project is the creation of a functional, packaged application, and its release marks the conclusion of the project. While any educational resource will require on-going evaluation to determine whether the objectives or projected outcomes have been achieved, it is considered essential to define a point at which the project is complete.

As each project Deliverable is being executed, it is associated with the influence level of the various Techniques and Skills specific to the development of interactive training materials. The $I^{3} \mathrm{D}$ model must be viewed as an integration of these three components, to ensure that all facets of interactive materials development are considered. More importantly, as the effective application of technology to enhance learning is better understood, so will the methods and strategies employed to develop the applications be refined. 


\section{Techniques}

The techniques for the development of interactive courseware are based on traditional ISD practices with one significant difference - each technique is perceived as active for the duration of the project, with the potential to influence the development of the product at any stage. Where the influence for a technique peaks represents the point at which it has most influence for the project, and embodied in each of these techniques are specific project activities which must be completed. However, unlike many ISD models which specify that activities should be completed at a particular stage of the Analysis or Design phase (e.g. identifying and describing the target population), the $I^{3} D$ model recommends that each activity has a major influence at particular stage, as well as the potential to continue to influence the project at any stage.

The functionality of the model is based on the flexibility of current development systems which allow modifications to be made to software (and therefore courseware) with little difficulty. Thus with each component of the techniques, the potential exists for influence on the project at any time during its execution, but with either increasing or decreasing impact. Thus information gathered from a research-related activity during the project can be catered for, but the closer to the end of the project, the less influence and impact it will or can have.

Another important attribute integral to the techniques (and skills), represented by the triangles, is that each may be considered dynamic, as illustrated in Figure 2, where the peak of influence of a technique or skill may be varied depending on the nature of the project. For example, a project identified as having significant levels of complex interactions may require more planning activities towards the beginning of the project, and an extension to this concept may be multiple influence-peaks, as shown in Figure 2(a). The dynamic nature of the $I^{3} D$ model therefore has the potential to be integrated into a formal project costing and estimation methodology. The following summary provides a brief description of the major elements in the Techniques component of the $I^{3} \mathrm{D}$ model:

\section{- Research}

This technique involves similar activities to those of the Analysis phase in traditional ISD methodologies, where information pertinent to the project is gathered and specifications relating to content, target audience and media are identified. However, 


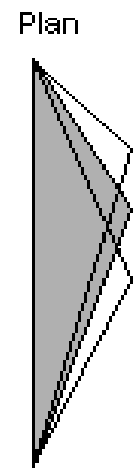

Figure 2

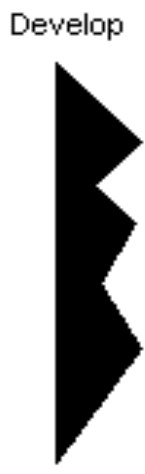

Figure 2a

research is considered a broader technique in that it will include an educational rationale for the project (including verification that technology is an appropriate delivery medium). Moreover, the concept that research outcomes may impact the project even during its latter stages has ramifications for the project manager in terms of enabling design or concept changes throughout the life of the project.

\section{- Planning}

Rather than identify Design as a specific technique, the notion of planning has been introduced to focus on the actual structuring of the instructional materials into a functional product, taking account of both the instructional and interactive components necessary for the success of a technology-based learning application. The design and strategies associated with this technique are considered a skill appropriate to the Interactive Architect.

\section{- Development}

The major technique for most projects is the development component, which includes the creation of all the materials associated with the product. However, rather than being dependent on the completion of previous phases (as in ISD methods), the model provides opportunity for development to be undertaken from the commencement of the project through to its completion. However, as indicated by the influence peak, development is likely to have major impact on the project in association with the Production deliverable.

\section{- Delivery}

Delivery and implementation issues typically have a major influence towards the end of the project, although concepts of delivery 
environments and administrative requirements will impact the overall project. As such, the concept of this technique having the potential to influence the project throughout its development is crucial to implementing the concepts of the $\mathrm{I}^{3} \mathrm{D}$ model.

\section{- Evaluation}

Rather than an activity enacted at the conclusion of the project, the $I^{3} \mathrm{D}$ model specifies evaluation as requiring constant influence, enabling regular monitoring of the progress and quality of the product. As evaluation is the key to success of learning resources, it will influence the entire project in at least three ways: (a) developmental, referring to strategies and criteria (or standards) applied during development to ensure functionality (does it work?) and applying "structured walkthroughs" to test logic and access; this would also include involvement by target group to assess navigation, control and interactivity, (b) formative, referring to final operational evaluation in the designated delivery environment and assessing whether the application functions according to specifications and that the stated learning objectives or outcomes are being addressed and (c) summative: normally undertaken some months after implementation to determine whether the overall outcomes have been achieved - has the original discrepancy between current and desired performance been resolved. Other issues relate to who is involved in the evaluation process, the timetable for evaluation and the means by which evaluation data will be used to revise the product.

\section{Skills}

The third component of the $\mathrm{I}^{3} \mathrm{D}$ model represents the Skills required for the development of effective interactive courseware. The most significant modification of the traditional skills identified is that the role of Instructional Designer has been split to include both a Learning Specialist as well as an Interactive Architect. As the success of interactive courseware depends on effective use of the medium, skills which combine screen design, human-computer interface, technology literacy and communication are required to ensure effective interactive strategies. In addition, the current impact of flexible and on-line learning demands the inclusion of skills in communications technology. Overall, these skills represent those required to work with the array of tools and technologies available today, and are summarised below: 


\section{- Learning Specialist}

This skill integrates an understanding of the ways in which people learn, the impact of research on individual differences in terms of developing software to support learning, the relationship between learning task and instructional techniques and the impact of educational philosophies and theories (for example, instructivism vs. constructivism) on the ways in which educational communication should be presented in a technological environment.

\section{- Interactive Architect}

In place of an Instructional Designer, the role and skill of the Interactive Architect is integral to the success and effectiveness of interactive courseware for teaching and learning. The skills associated with an interactive architect focus on those elements of instruction and learning which are best suited to the interactive environment, i.e. those which keep the learner involved and engaged for the duration of the lesson (Sims, 1997). This skill also supports the notion that interactive technology is an emerging medium, and that new skills are necessary to implement effective applications. It is this new range of skills which will enable the ultimate realisation of the assumptions identified in the first part of the discussion.

\section{- Graphics and media}

With the advent of sophisticated graphics, animation, audio and video, skills in both media and graphics are essential for interactive training applications. Within the model, the range of skills (and their relative influence) are included under the same component. However, it is recognised that the growing level of specialisation in multimedia production might necessitate the separation of this skill into separate categories for project success.

\section{- Software development}

This is a vital skill which may include the use of authoring tools, and which definitely requires programming ability. While easy-to-use development tools are often promoted, they can result in simply structured courseware, and without interaction and branching, the courseware is unlikely to be more than an information presentation system. The ability to take advantage of the processing power of the technology through the application of programming techniques is an essential skill for developing effective interactive learning products. 


\section{- Communications technology}

As on-line learning is set to become the new benchmark for teaching institutions, the ability to take advantage of a technological system based on information transfer via networks will require expertise in the network protocol and operation. While the advent of the internet and World Wide Web does not imply on-line learning will be better (as discussed previously), enabling effective communications through the network will likely be an essential component of interactive learning and therefore technical network-related skills are integral to such projects.

\section{- Content specialist}

The development of interactive training also requires skills and expertise in the content area. It is essential that individuals identified with these skills are recognised as experts in the field, and familiar with the application of the content in the work environment. This skill is perceived as having equal influence throughout the project.

\section{- Project control}

A courseware development activity requires skills in project control and management, which also have influence throughout the project. While traditional project management skills are useful, there is also the demand for understanding that interactive projects have greater potential for modification and change throughout the duration of the project, as determined by the notion of influence of the techniques and their potential for impact throughout the project. By maintaining control over quality while catering for levels of influence on development activities, the project manager will play a significant role in the successful production of effective interactive learning materials.

\section{Summary}

The development of the $I^{3} \mathrm{D}$ model is important because it represents an attempt to provide a framework for the technologies available to current developers. Through integrating and linking the Deliverables, Techniques and Skills of courseware development, it is proposed that there is greater likelihood of developing effective interactive learning materials. By introducing the notion of influence, a more realistic representation of the role human factors and change is provided for. 


\section{Conclusions}

When given the opportunity to discuss emerging technologies, the urge is to unveil applications of the latest and greatest technologies. In the late 1990 's, these technologies almost exclusively revolve around multimedia and on-line learning. And with these technologies, the temptation is to predict the extent to which they will provide the solution to educational and performance technology.

This paper contends however, that technology, although complex and invaluable as a business tool, may not be as flexible as many established educational resources - such as the book or classroom tutorial. Rather than identify the means by which technology could replicate these resources, it is suggested that extensive research is required to determine what technology does best and how it can be manipulated to provide effective interactive learning environments. Through the introduction of an enhanced development and control methodology which integrates all elements of courseware development (instructional design, personnel and resources), a realistic environment is provided for the production of quality courseware potentially better, cheaper and faster.

Interactive learning and performance support is an emerging technology. Its future success depends on recognising this condition and developing strategies and techniques which take advantage of the technology.

\section{References}

Alessi, S. \& Trollip, S. (1991). Computer-Based Instruction: Methods and Development. 2nd Edition. Englewood Cliffs, NJ: Prentice Hall.

Allen, M. (1992). Successive Approximations and Courseware Development. Paper presented at the 10th Annual Computer-Based Training Conference. Orlando, Florida, 1992.

Aubrey, J. (1992). The interactive instructional influence model. Paper presented as part of the requirements for completion of the Graduate Diploma in Computer Based Learning. University of Technology, Sydney.

Clark, R.C. (1983). Reconsidering research on learning from media. Review of Educational Research, 53(4), 415-419.

DeWeaver, M.F. \& Gillespie, L.C. (1997). Real World Project Management: New Approaches for Adapting to Change and Uncertainty. Quality Resources.

Foshay, R. (1997). Posting to the ITFORUM Listserver, March. ITFORUM@UGA.CC.UGA.EDU. 
Gillespie, L., Sims, R. \& Spannaus, T. (1992). ISD on trial. General Session held at the 34th Annual ADCIS Conference. Norfolk, VA.

Gustafson, K.L. (1991). Survey of Instructional Development Models. Syracuse University: ERIC.

Jonassen, D.H. (Ed) (1988). Instructional Designs for Microcomputer Courseware. Hillsdale, NJ: Lawrence Erlbaum Associates.

Kulik, D., Kulik, J. \& Cohen, P. (1980). Instructional technology and college teaching. Teaching of Psychology, 7, 199-205.

Larsen, R.E. (1992). Relationship of learning style to the effectiveness and acceptance of interactive video instruction. Journal of Computer-Based Instruction, 19(1), 17-21.

Leshin, C.B., Pollock, J. \& Reigeluth, C.M. (1992). Instructional Design Strategies and Tactics. Englewood Cliffs, NJ: Educational Technology Publications.

Logan, R.S. (1979). A State-of-the-Art Assessment of Instructional Systems Development. In H. F. O'Neil (Ed), Issues in Instructional Systems Development. New York: Academic Press.

Merrill, M.D. (1997). Instructional Transaction Theory (ITT): Instructional design based on knowledge objects. Posting to the ITFORUM Listserver, September 19. ITFORUM@UGA.CC.UGA.EDU and http://www.coe.usu.edu/coe/id2/ddc0997.html.

Merrill, M.D., Li, Z. \& Jones, M.K. (1990). Second generation instructional design $\left(\mathrm{ID}_{2}\right)$. Educational Technology, 30(1), 7-11.

Merrill, M.D., Li, Z. \& Jones, M.K. (1991). Instructional Transaction Theory: An introduction. Educational Technology, 31(6), 7-12.

Phillips, R. (Ed) (1996). Developers Guide to Interactive Multimedia. Perth, WA: Curtin University of Technology.

Reeves, T.C. (1993). Pseudoscience in computer-based instruction: The case of learner-control research. Journal of Computer-Based Instruction, 20(2), 39-46.

Roblyer, M.D. (1988). Fundamental Problems and Principles of Designing Effective Courseware. In D. H. Jonassen (Ed), Instructional Designs for Microcomputer Courseware. Hillsdale, NJ: Lawrence Erlbaum Associates.

Ross, S.M. \& Morrison, G.R. (1988). Adapting Instruction to Learner performance and Background Variables. In D. H. Jonassen (Ed), Instructional Designs for Microcomputer Courseware. Hillsdale, NJ: Lawrence Erlbaum Associates.

Sims, R. (1990). Towards an Individualised Learner-Computer Interface. In A. McDougall \& C. Dowling (Eds), Computers in Education. Proceedings of the IFIP TC3 5th World Conference on Computers and Education. Amsterdam: Elsevier.

Sims, R. (1992). Meta-CBT II: The final product. Proceedings of the 34th ADCIS International Conference. Norfolk, VA: ADCIS. 
Sims, R. (1996). Engagement, control and the learner: A theoretical appraisal of interactivity. In A. Christie, P. James \& B. Vaughan (Eds), Making new connections. Proceedings of the 13th Annual Conference of the Australian Society for Computers in Learning in tertiary Education (ASCILITE). Adelaide, SA: University of South Australia.

Sims, R. (1997). Interactivity: A forgotten art? Computers in Human Behavior, 13(2), 157-180.

Sims, R. \& Schwalger, S . (1992). Competency-Based Training: Management Systems and Interactive Courseware. In A. Holzl \& D. Robb (Eds), Proceedings of the 2nd International Conference for Information Technology in Training and Education (ITTE'92). Brisbane: University of Queensland.

Tessmer, M. \& Richey, R.C. (1997). The role of context in learning and instructional design. Educational Technology Research and Development, 45(2), 85-115.

The Australian (1997). Online to cheap learning. Wednesday, October 1, 47.

Trollip, S.R. (1992). Testing: The forgotten component of CAI. In B. Chia, R. Pennell \& R. Sims (Eds), A Future Promised. Proceedings of the 9th Conference of the Australian Society for Computers in Learning in Tertiary Education (ASCILITE'92). Sydney: ASCILITE.

Viers, G.R. (1996). Posting to the ITFORUM Listserver, October 23. ITFORUM@UGA.CC.UGA.EDU.

Yang, C., Moore, D.M. \& Burton, J.K. (1995). Managing courseware production: An instructional design model with a software engineering approach. Educational Technology Research and Development, 43(4), 60-70.

Roderick C.H. Sims

School of Multimedia \& Information Technology

Southern Cross University

Coffs Harbour, NSW Australia

rsims@scu.edu.au 